\title{
Critérios de avaliação da qualidade da informação em sites de saúde: uma proposta
}

\author{
Criteria to evaluate quality of information on health sites: a proposal \\ Los criterios de evaluación de calidad de la información en sitios web de \\ salud: una propuesta.
}

\begin{abstract}
Ana Paula Bernardo Mendonça| anapaulamendonca@fiocruz.br
Fundação Oswaldo Cruz, Escola Nacional de Saúde Pública Sergio Arouca, Programa de Pós Graduação em Saúde Pública de : Mestrado Profissional em Política e Gestão de Ciência, Tecnologia e Inovação em Saúde (CT\&I). Rio de Janeiro, RJ, Brasil.
\end{abstract}

\section{André Pereira Neto | apereira@fiocruz.br}

Fundação Oswaldo Cruz, Escola Nacional de Saúde Pública Sergio Arouca, Programa de Pós Graduação em Saúde Pública: Mestrado Profissional em Política e Gestão de Ciência, Tecnologia e Inovação em Saúde (CT\&I). Rio de Janeiro, RJ, Brasil.

\section{Resumo}

A internet oferece ao cidadão uma quantidade incomensurável de informações em todas as áreas do conhecimento. Elas são disponibilizadas sem que haja qualquer tipo avaliação. Na saúde, essas informações podem prejudicar o cidadão. O usuário precisa compreender o que encontra na rede e acreditar no que lê. A avaliação da qualidade das informações sobre saúde encontradas na internet é um problema que muitas instituições e pesquisadores têm procurado resolver. Este artigo tem como objetivo apresentar alguns critérios de avaliação da qualidade das informações encontradas em sites de saúde. Para isso, foi realizada uma pesquisa de modo a identificar os principais instrumentos nacionais e internacionais que têm essa finalidade, foram analisados seus métodos de aplicação e comparados os critérios adotados. Como resultado, oitenta critérios de qualidade de sites de saúde foram agrupados em três dimensões: técnica, de conteúdo e design. Recomenda-se a criação de um selo de qualidade para sites de saúde no Brasil.

Palavras-chave: Internet; Informação de saúde ao cidadão; Certificação; Avaliação.

\section{Abstract}

The internet provides citizens with an immeasurable amount of information in all areas of knowledge. They are available without any evaluation. In health, this information may harm the citizen. The users must understand what they find online and believe that the information is true. The evaluation of quality of health information found on the internet is a problem that many institutions and researchers are seeking to solve. This article aims to present some criteria to evaluate the quality of information found on health 
sites. A research was conducted to identify the main national and international instruments that have this purpose, then we have analyzed its application methods and compared the criteria adopted. As result eighty quality criteria of health websites were grouped into three dimensions: technical, content and design. We recommend that the health sites in Brazil can be certified by the development of a seal of quality.

Keywords: Internet; Health information to citizen; Certification; Evaluation.

\section{Resumen}

Internet ofrece a los ciudadanos una cantidad inconmensurable de informaciones en todas las áreas del conocimiento. Están disponibles sin ningún tipo de evaluación. En materia de salud, esas informaciones pueden hacer daño al ciudadano. El usuario debe entender lo que está en la red y creer que las informaciones son verdaderas. La evaluación de la calidad de las informaciones acerca de la salud que están en internet es un problema que muchas instituciones y investigadores procuran resolver. Este artículo tiene el objetivo de presentar algunos criterios de evaluación de la calidad de las informaciones encontradas en sitios web de salud. Realizamos una investigación para identificar los principales instrumentos nacionales e internacionales que tienen este propósito y, después, analizamos sus métodos de aplicación y comparamos los criterios adoptados. Como resultado, ochenta criterios de calidad para sitios web de salud fueron agrupados en tres dimensiones: técnica, contenido y diseño. Recomendamos la creación de un sello de calidad para los sitios de salud en Brasil.

Palabras clave: Internet; Información de salud al ciudadano; Certificación; Evaluación.

Contribuição dos autores: Os dois autores participaram no levantamento bibliográfico, da pesquisa empírica e da redação do manuscrito.

Declaração de conflito de interesses: Não há conflito de interesses.

Fontes de financiamento: Não houve fonte de financiamento.

Considerações éticas: Não procede, pois os documentos analisados são de domínio público.

Histórico do artigo: Submetido: 22.jan.2015 | Aceito: 18.fev.2015 | Publicado: 31.mar.2015

Apresentação anterior: Mendonça APB. Critérios de avaliação de qualidade para sites de saúde: uma proposta. [dissertação de mestrado]: Rio de Janeiro: Fundação Oswaldo Cruz, Escola Nacional emde Saúde Pública Sergio Arouca;, 2013. 98 p. Programa de Pós Graduação de Saúde Pública: Mestrado Profissional em Política e Gestão de Ciência, Tecnologia e Inovação em Saúde (CT\&I); 2013. [citado 23 mar 2015]. Disponível em: http://157.86.8.70:8080/certifica/bitstream/icict/2393/2/0000108.pdf

Mendonça APB. Critérios de avaliação de qualidade para sites de saúde: uma proposta. [aApresentação da dDissertação de mestrado]: Rio de Janeiro: Fundação Oswaldo Cruz, Escola Nacional de Saúde Pública Sergio Arouca;, 2013. 24 p. Programa de Pós Graduação em de Saúde Pública: Mestrado Profissional em Política e Gestão de Ciência, Tecnologia e Inovação em Saúde (CT\&I); 2013. [citado 23 mar 2015]. Disponível em http://pt.slideshare.net/anapaulabm/defesa-1607final

Licença CC BY-NC atribuição não comercial. Com essa licença é permitido acessar, baixar (download), copiar, imprimir, compartilhar, reutilizar e distribuir os artigos, desde que para uso não comercial e com a citação da fonte, conferindo os devidos créditos de autoria e menção à Reciis. Nesses casos, nenhuma permissão é necessária por parte dos autores ou dos editores. 


\section{Introdução}

A internet é o meio pelo qual um grande número de pessoas ao redor do mundo busca informações diversas ${ }^{1}$. A saúde desponta como uma das áreas sobre a qual há cada vez mais informação disponível em sites e páginas de instituições públicas e privadas². Muitas informações estão disponíveis também em ambientes coletivos virtuais nos quais se prolifera a troca de experiências entre pacientes, profissionais e organizações que prestam serviços de saúde 3 . Essas informações abrangem, muitas vezes, a prevenção, o diagnóstico e o tratamento de doenças ${ }^{4}$. O interesse por elas é cada vez maior e se justifica na medida em que os assuntos relacionados à saúde representam uma das principais preocupações do homem contemporâneo ${ }^{5}$.

Segundo Moraes ${ }^{6}$, informação em saúde é toda informação relacionada com as condições de vida e morte de indivíduos e populações. Incluem-se, ainda, aquelas informações sobre comportamentos, produtos e serviços relacionados com o corpo e a saúde.

A quantidade de informação online sobre temas vinculados, de alguma forma, às questões relativas à saúde cresce vertiginosamente. Uma busca no Google, realizada em janeiro de 2014 utilizando o termo "tuberculose", recuperou cerca de 7.760.00o registros em língua portuguesa. Eles têm diferentes origens, características, finalidades, temas e teores. Tal achado reflete a natureza heterogênea das informações sobre saúde recuperadas na internet, encontradas em ambientes que vão desde sites (jornais, educacionais, acadêmicos, hospitais, clínicas e consultórios) até grupos de autoajuda organizados por indivíduos diagnosticados com determinada condição e, ainda, comunidades virtuais, redes sociais e fóruns de discussão.

Nenhum desses ambientes virtuais sofre(u) qualquer avaliação antes de ser disponibilizado na internet. As informações que contêm podem estar incompletas, incorretas ou serem incompreensíveis para um cidadão comum. Diante desta realidade, esse último tem dificuldade de distinguir o certo do enganoso ou o inédito do tradicional7.

Considerando os riscos que informações encontradas na internet oferecem, especialistas em informação vêm desenvolvendo checklists contendo critérios e indicadores na tentativa de estabelecer padrões mínimos de qualidade ${ }^{8}$.

Segundo Fogg e colaboradores ${ }^{9}$, alguns indicadores ampliam enquanto outros diminuem a qualidade da informação na web. Um deles está relacionado com o domínio do site. Se ele for governamental (.gov), educacional (.edu) ou institucional (.org) terá melhor reconhecimento do que se ele for pessoal. Ele também terá boa aceitação se contiver o endereço que permita que o usuário entre em contato com o gestor da página e tenha suas dúvidas atendidas ${ }^{9}$. Para Mostafa e Terra $^{10}$, grande parte dos critérios obedece a um consenso sobre os questionamentos básicos relacionados com o processo de comunicação científica, ou seja, valorizam a relevância, a autoridade, a objetividade, a atualização e a cobertura do site.

Eysenbach e colaboradore ${ }^{11}$ publicaram, em 2002, o resultado de uma revisão sistemática analisando 79 estudos que avaliaram a qualidade da informação em sites de saúde. Eles apresentaram cinco critérios de qualidade mais frequentemente utilizados, a saber: Técnico - refere-se à questão de como a informação foi obtida e disponibilizada. São variações do que poderia ser chamado de critério de credibilidade, transparência e ética profissional; Acurácia - mensura o grau de concordância da informação fornecida com a melhor evidência ou com a prática médica; Abrangência - observa o grau de cobertura da informação; Intelegibilidade - verifica o nível de complexidade e dificuldade de leitura do texto e Design - avalia aspectos visuais do site.

Risk e Dzenowagis ${ }^{12}$ identificaram iniciativas de organizações internacionais de avaliação da qualidade da informação em saúde apresentada na internet. Elas estão concentradas nos países desenvolvidos da Europa e nos Estados Unidos. Para estes autores, mesmo quando a informação apresentada for de alta qualidade, poderá oferecer riscos aos cidadãos. Ela pode oferecer questões incompreensíveis devido à 
complexidade da linguagem e/ou à não adequação ao público leigo, à dificuldade de interpretação de dados científicos, à imprecisão e/ou à falta de atualização das informações. Além disso, os interesses comerciais devem ser considerados.

O objetivo deste artigo é apresentar uma proposta de critérios e indicadores de avaliação de qualidade para sites de saúde. Para tanto, foram identificadas as iniciativas internacionais de avaliação de qualidade de sites de saúde e selecionados os critérios de qualidade mais utilizados.

\section{Metodologia}

A metodologia adotada neste estudo focalizou quatro objetivos específicos: identificar os estudos voltados para avaliação das informações em saúde disponíveis na internet; identificar iniciativas nacionais e internacionais de avaliação da qualidade das informações de saúde na web; relacionar os critérios de qualidade que foram utilizados por essas iniciativas; identificar os métodos de aplicação desses critérios; selecionar critérios a serem utilizados em sites de saúde no Brasil. Para atender estes objetivos dividimos nosso trabalho em três etapas:

\section{Etapa 1. Pesquisa bibliográfica}

Para identificar os estudos voltados para avaliação das informações em saúde disponíveis na internet, realizou-se uma busca de artigos científicos na Scientific Electronic Library Online (Scielo) e na Biblioteca Virtual de Saúde Pública (BVS). Foram utilizadas as palavras de busca sites, avaliação e saúde. Utilizamos como filtro o idioma português. Foram lidos o título e o resumo. Assim, foi avaliada a pertinência de cada título selecionado. Como critério de inclusão, selecionamos trabalhos que tratassem especificamente de avaliar a qualidade da informação sobre saúde disponível em sites. Assim, foram selecionados os seguintes estudos: Silva, Mello e Mion ${ }^{4}$, Souza, Luz e Rabello ${ }^{13}$, Lopes $^{14-15}$, Leite e Correia ${ }^{16}$, Silva e Gubert ${ }^{17}$, Malafaia et al. ${ }^{18}$, Silveira, Costa e Lima ${ }^{19}$ e Cubas e Felchner ${ }^{2}$. A partir da análise das referências bibliográficas destes estudos, foram localizados trabalhos de Silberg, Lundberg e Musacchio ${ }^{20}$, Jadad e Gagliardi ${ }^{21}$, Gagliardi e Jadad $^{22}$, Risk e Dzenowagis ${ }^{12}$ e Eysenbach et al $^{11}$. Lopes ${ }^{14}$ publicou um livro em que relaciona as iniciativas internacionais e nacionais protagonistas na área de regulação e avaliação da qualidade de informação em saúde na web.

\section{Etapa 2. Iniciativas de avaliação da qualidade da informação}

Risk \& Dzenowagis ${ }^{12}$ e Lopes ${ }^{14}$ analisaram as iniciativas de avaliação da qualidade da informação em sites de saúde promovidas por instituições públicas e privadas no mundo. Devido ao caráter dinâmico da web e o ano de publicação das pesquisas nos sentimos obrigados a visitar o site de cada organização relacionada a fim de verificar suas respectivas atualizações. Para análise dos critérios utilizados por tais instituições, elaborou-se uma síntese das organizações pertinentes, relacionando seus instrumentos ou processos de avaliação de acordo com as suas principais características.

\section{Etapa 3. Critérios de qualidade e composição da proposta de critérios de qualidade para sites de saúde}

Para melhor entender a relação entre os critérios e agrupá-los em dimensões de avaliação da qualidade, identificamos as possíveis similaridades, complementaridades e divergências entre as iniciativas consideradas mais relevantes. Por fim, agrupamos os critérios em dimensões relativas à qualidade. Com isso, fomos capazes de fazer uma proposição para o adequado atendimento do objetivo da pesquisa em pauta. 


\section{Resultados e discussão}

As iniciativas identificadas podem ser agrupadas, segundo os instrumentos de avaliação da qualidade da informação que elas propõem, em três tipos: "códigos de conduta"; "guias de usuários" e "certificação de qualidade da informação na web”.

Os "códigos de conduta" apresentam uma lista de categorias e subcategorias de problemas críticos relacionados com a qualidade da informação na web. Funcionam como guia para os provedores de informação em saúde, norteando a elaboração de sites. Eles sugerem padrões recomendados por especialistas que podem assegurar maior credibilidade às informações disponibilizadas ${ }^{14}$. Eles se baseiam na honestidade do provedor da informação e, portanto, podem ser aplicados sem a verificação por parte da organização criadora. Isto significa que seu cumprimento é voluntário. Neste caso, na medida em que não há mecanismos de verificação por terceiros, não há garantia de que os sites cumpram as regras de qualidade sugeridas ${ }^{12}$.

Os "guias de usuários" são uma maneira de aplicar um "código de conduta". Ele assume a forma questionário de verificação (checklist). Neste caso, a avaliação é realizada através de formulário eletrônico baseado em critérios estabelecidos por um código de ética ou código de conduta. O formulário é apresentado para o usuário verificar a conformidade entre a informação disponibilizada e os critérios adotados. O principal objetivo deste instrumento é estimular o usuário a julgar a qualidade da informação que lhe é relevante de acordo com os critérios oferecidos. Este processo apresenta baixo custo de implementação e possibilita ao provedor de informação saber o nível de satisfação dos usuários sobre a informação disponibilizada ${ }^{12}$. Seguindo o modelo do "código de conduta", os "guias de usuários" transferem grande responsabilidade para os usuários que, em geral, não estão devidamente qualificados para realizar essa avaliação. O sucesso dessa iniciativa envolve um grande trabalho de divulgação de sua importância, assim como implica a existência de uma estrutura de suporte para o atendimento ao usuário em caso de dificuldade de utilização $0^{12}$.

A "certificação de qualidade" da informação na web é um processo que atribui uma logomarca ou selo que identifica que o site em questão atende aos critérios verificados pela instituição credenciadora. "Trata-se de recebimento de selos de certificação através da adoção voluntária ou obrigatória de um código de ética que regulamenta o conteúdo informacional disponibilizado e os serviços prestados por sites institucionais, comerciais e pessoais" ${ }^{23}$.

Os sistemas de certificação ou acreditação de qualidade da informação constituem-se de um rigoroso processo de análise de vários aspectos entre os quais se destaca a participação de especialistas que garantem qualidade de informação de um site ${ }^{24}$. A certificação de um site possui um custo alto, pois requer uma equipe multidisciplinar de especialistas. Ela apresenta, entretanto, uma vantagem em relação aos "guias de usuários" e aos "códigos de conduta": os sistemas de certificação ou acreditação de qualidade da informação transferem a responsabilidade da avaliação para as organizações credenciadoras ${ }^{25}$.

Os Estados Unidos contam com o maior número de iniciativas na área. Uma das mais tradicionais é a Agency for Healthcare Research and Quality (AHRQ) ${ }^{26}$. Ela atua nessa área desde 1997. Sua missão é produzir evidências para tornar o cuidado em saúde equitativo, mais seguro e acessível e com melhor qualidade através do Departamento de Saúde e Serviços Humanos dos Estados Unidos. A AHRQ promulgou, em 2001, o documento intitulado Criteria for Assessing the Quality of Health Information on the Internet. Nele, foram estabelecidos critérios para avaliar a qualidade da informação sobre saúde na internet.

A American Medical Association (AMA) desenvolveu normas de qualidade com o objetivo de manter o controle de qualidade dos sites de seus associados publicou o Guidelines for medical and health information sites on the Internet ${ }^{27}$ ou AMA Guidelines, estabelecido por meio de um consenso entre os especialistas, autoridades e usuários ${ }^{25}$. O guia foi concebido para orientar os responsáveis pelo desenvolvimento de sites dos associados. Os critérios da AMA correspondem quase integralmente aos critérios desenvolvidos pela AHRQ ${ }^{26}$. 
A Internet Healthcare Coalition (IHC) ${ }^{28}$ é uma organização sem fins lucrativos que reúne seguradoras de saúde nos Estados Unidos (EUA) desde 1997. Sua função principal é fornecer alternativas acessíveis de cuidados de saúde pela internet. Em 2000, a IHC elaborou o código de ética no qual apresenta oito princípios para sites de saúde. O eHealth Code of Ethics visa garantir que pessoas possam confiar na informação disponível e tenham plena compreensão dos riscos inerentes à adoção desta ou daquela prática. O IHC percebe o potencial da internet na gestão da saúde do cidadão por meio do seu cuidado.

A Accreditation Healthcare Commission, organização sem fins lucrativos fundada em 1990, tem como missão melhorar a qualidade e eficiência da gestão dos cuidados de saúde através de processos de credenciamento e educação. Ela criou em 2001, o sistema de credenciamento de sites denominado Utilization Review Accreditation Commission (URAC) ${ }^{29}$. Ele verifica cerca de 50 itens de qualidade para credenciar sites de saúde. O processo de acreditação é iniciado, após a solicitação do requerente, por um comitê de acreditação e revisão, que inclui especialistas de diversas áreas. Um requisito importante deste programa de acreditação é que o requerente deve demonstrar que estabeleceu um comitê de qualidade para supervisionar anualmente a conformidade com os padrões ${ }^{25}$.

Na Europa, destaca-se a Comissão da Comunidade Europeia. Esta organização continental publicou o documento intitulado Health Literacy and Internet. Recommendations to promote Health Literacy by the means of the Internet ${ }^{30}$ em que estabelece um conjunto de critérios de qualidade para uso dos Estados membros e para o público em geral. O objetivo principal foi orientar autoridades nacionais e regionais, associações de profissionais de saúde e instituições que disponibilizam suas páginas na web a seguirem os critérios de qualidade propostos.

Em relação à orientação aos usuários, duas iniciativas europeias chamam mais a atenção: uma inglesa e outra francesa. Em 1999, o Instituto de Ciências da Saúde na Inglaterra da British Library \& University Oxford criou o DISCERN ${ }^{31}$. Ele é composto de um formulário online com 15 questões. Elas estão organizadas em três seções, desenvolvidas especialmente para que os indivíduos que procuram informação sobre tratamentos avaliem se a informação disponível no site consultado é ou não confiável e abrangente ${ }^{31}$.

Além disso, existem outras iniciativas na Inglaterra, França e Espanha.

A associação francesa "Centrale Santé" ${ }^{2}$ inspirada no "Código de Ética da Internet Healthcare Coalition" (IHC), elaborou o NET Scoring, incorporando alguns aspectos relacionados com a comunidade francesa no contexto europeu. Para garantir a máxima objetividade na seleção dos critérios, a associação formou um grupo multidisciplinar que incluiu profissionais de saúde, engenheiros, bibliotecários, advogados e representantes de algumas organizações profissionais que definiram questionário com 49 critérios divididos em oito categorias. Cada critério recebe um peso específico, a saber: critério essencial (peso de zero a nove); critério importante (peso de zero a seis) e critério menor (peso de zero a três). O total desses critérios atribuído a uma página web fornece a respectiva medida de desempenho dessa página ${ }^{14}$.

A Fundação Health On the Net (HON), sediada na Suíça, é uma da mais antigas e conhecidas iniciativas de avaliação da qualidade da informação sobre saúde na internet. A HON atribui um selo de qualidade para os sites de saúde que estejam em conformidade com os princípios do HONCode ${ }^{33}$. Para muitos autores, o HONCode é considerado como "padrão ouro" em termos de certificação de informação em sites de saúde ${ }^{14}$. Além disso, ele possui o maior número de sites acreditados no mundo. O selo HONCode pode ser encontrado em mais de 8.300 sites sobre saúde, de 102 países em 35 idiomas. Todos os sites certificados são revistos, anualmente, para ratificar ou não o credenciamento em atendimento aos princípios estabelecidos. A instituição fornece ainda um código de conduta para orientar os usuários a avaliarem se um determinado site está em conformidade com seus princípios. Para tanto, dispõe de um vídeo instrucional para ajudar aos usuários identificar sites confiáveis.

Na Espanha, a "Web Mèdica Acreditada” (WMA) ${ }^{34}$, programa de certificação vinculado ao Colegio Oficial de Médicos de Barcelona (COMB), oferece um selo de qualidade que identifica os sites que atendem 
a determinados requisitos em termos de qualidade de conteúdo e confiabilidade dentro da indústria médica e de saúde da Espanha. Outra iniciativa espanhola vem da Andaluzia. Trata-se da Agencia de Calidad Sanitaria de Andalucía. Ela desenvolveu o Programa de acreditación de páginas web sanitarias. Baseado nas recomendações da iniciativa da Comissão Europeia, este programa pretende verificar o nível de confiança que as páginas web têm em relação ao conteúdo, conectividade e aos serviços que oferecem aos seus usuários. A agência também propõe um selo de confiança, concedido por meio do mencionado programa, que define diferentes níveis de acreditação, a saber: avançado, bom ou excelente. Estes níveis indicam o grau de conformidade com os grupos de critérios atendidos. A validade do selo atribuído ao site avaliado é de dois anos. Após esse período, o gerente do site terá que submetê-lo para a certificação novamente.

No Brasil, é digna de nota a iniciativa do Conselho Regional de Medicina de São Paulo (Cremesp). Esta entidade médica elaborou o Guia de ética para sites de medicina e saúde na internet ${ }^{35}$ em que define princípios éticos e estabelece padrões mínimos de qualidade, segurança e confiabilidade para sites de medicina e saúde. Médicos e instituições de saúde registrados no Cremesp passaram a ficar obrigados a adotar esse guia para efeito de idealização, registro, criação, manutenção, colaboração e atuação profissional em domínios, sites, páginas, ou portais sobre medicina e saúde na internet. Esses princípios podem ser considerados norteadores para a construção uma política de autorregulamentação e de conduta de sites de saúde na internet.

Os instrumentos de avaliação que apresentamos, aqui, foram criados há mais de dez anos. Em sua maioria, foram publicados somente em um idioma. A oferta de versões em mais de um idioma aumentaria o alcance e a disseminação de boas práticas para melhoria da qualidade da informação online. Considerando que as transformações tecnológicas provocam constantes mudanças no próprio ambiente da web, tais instrumentos precisam ser constantemente reavaliados e readaptados ${ }^{14}$.

Os aspectos mais abordados nas iniciativas de avaliação mencionadas acima estão relacionados com a autoria, periodicidade de atualização e apresentação das fontes da informação. Apesar da semelhança entre os critérios dos códigos analisados, percebemos uma ausência de padronização das terminologias empregadas aos critérios. Com base nos dados disponíveis construímos o seguinte quadro comparativo:

Quadro 1 - Instrumentos de avaliação

\begin{tabular}{|c|c|c|c|c|c|c|}
\hline Ano & Instrumento & País & Idioma & Público & Métodos & Princípios éticos/critérios \\
\hline 1996 & HONCode & Suíça & 35 idiomas & $\begin{array}{l}\text { Usuários } \\
\text { Provedores de } \\
\text { informação }\end{array}$ & $\begin{array}{l}\text { Código de } \\
\text { conduta } \\
\text { Certificação } \\
\text { Guia de } \\
\text { usuários }\end{array}$ & $\begin{array}{l}\text { Autoridade, } \\
\text { complementaridade, } \\
\text { confidencialidade, atribuições, } \\
\text { justificativas, transparência } \\
\text { na propriedade, transparência } \\
\text { do patrocínio, honestidade } \\
\text { da publicidade e da política } \\
\text { editorial. }\end{array}$ \\
\hline 1997 & Discern & $\begin{array}{l}\text { Reino } \\
\text { Unido }\end{array}$ & Inglês & $\begin{array}{l}\text { Usuários } \\
\text { Provedores de } \\
\text { informação }\end{array}$ & $\begin{array}{l}\text { Guia de } \\
\text { usuários }\end{array}$ & $\begin{array}{l}\text { Confiabilidade da publicação, } \\
\text { abrangência sobre opções de } \\
\text { tratamento e qualidade da } \\
\text { informação em geral no site. }\end{array}$ \\
\hline 1997 & NET Scoring & França & $\begin{array}{l}\text { Inglês } \\
\text { Francês }\end{array}$ & $\begin{array}{l}\text { Usuários } \\
\text { Provedores de } \\
\text { informação }\end{array}$ & $\begin{array}{l}\text { Guia de } \\
\text { usuários }\end{array}$ & $\begin{array}{l}\text { Credibilidade, conteúdo, } \\
\text { links, design, interatividade, } \\
\text { aspectos quantitativos, ética e } \\
\text { acessibilidade. }\end{array}$ \\
\hline
\end{tabular}




\begin{tabular}{|c|c|c|c|c|c|c|}
\hline Ano & Instrumento & País & Idioma & Público & Métodos & Princípios éticos/critérios \\
\hline 1997 & $\begin{array}{l}\text { Criteria for } \\
\text { Assessing } \\
\text { the Quality } \\
\text { of Health } \\
\text { Information on } \\
\text { the Internet }\end{array}$ & EUA & Inglês & $\begin{array}{l}\text { Usuários } \\
\text { Provedores de } \\
\text { informação }\end{array}$ & $\begin{array}{l}\text { Código de } \\
\text { conduta }\end{array}$ & $\begin{array}{l}\text { Credibilidade, conteúdo, } \\
\text { apresentação formal do site, } \\
\text { links, design, interatividade e } \\
\text { anúncios. }\end{array}$ \\
\hline 1999 & $\begin{array}{l}\text { WMA Sello de } \\
\text { calidad }\end{array}$ & Espanha & Espanhol & $\begin{array}{l}\text { Sites que } \\
\text { desejam obter } \\
\text { um selo de } \\
\text { qualidade }\end{array}$ & Certificação & $\begin{array}{l}\text { Conteúdo científico, autoria, } \\
\text { atualização do conteúdo, } \\
\text { fontes da informação, aspectos } \\
\text { éticos, legais e profissionais, } \\
\text { características básicas de } \\
\text { usabilidade. }\end{array}$ \\
\hline 2000 & $\begin{array}{l}\text { AMA } \\
\text { Guidelines }\end{array}$ & EUA & Inglês & $\begin{array}{l}\text { Sites da } \\
\text { associação }\end{array}$ & $\begin{array}{l}\text { Código de } \\
\text { conduta }\end{array}$ & $\begin{array}{l}\text { Conteúdo, publicidade e } \\
\text { patrocínio, privacidade e } \\
\text { confidencialidade e comercio } \\
\text { eletrônico. }\end{array}$ \\
\hline 2000 & $\begin{array}{l}\text { e-Health Code } \\
\text { of Ethics }\end{array}$ & EUA & Inglês & $\begin{array}{l}\text { Usuários } \\
\text { Provedores de } \\
\text { informação }\end{array}$ & $\begin{array}{l}\text { Código de } \\
\text { conduta }\end{array}$ & $\begin{array}{l}\text { Imparcialidade; honestidade, } \\
\text { qualidade, consentimento } \\
\text { sobre uso de dados pessoais, } \\
\text { privacidade, profissionalismo } \\
\text { na atenção à saúde online, } \\
\text { parceria responsável e } \\
\text { fiscalização no cumprimento do } \\
\text { código. }\end{array}$ \\
\hline 2001 & URAC Seal & EUA & Inglês & $\begin{array}{l}\text { Sites que } \\
\text { desejam obter } \\
\text { um selo de } \\
\text { qualidade }\end{array}$ & Certificação & Apresenta 53 critérios. \\
\hline 2002 & $\begin{array}{l}\text { Quality } \\
\text { Critéria for } \\
\text { Health related } \\
\text { Websites }\end{array}$ & $\begin{array}{l}\text { União } \\
\text { Européia }\end{array}$ & 11 idiomas & $\begin{array}{l}\text { Sites que } \\
\text { desejam obter } \\
\text { um selo de } \\
\text { qualidade }\end{array}$ & $\begin{array}{l}\text { Código de } \\
\text { conduta }\end{array}$ & $\begin{array}{l}\text { Transparência e honestidade, } \\
\text { autoridade, privacidade } \\
\text { e proteção dos dados } \\
\text { pessoais, atualização das } \\
\text { informações, responsabilidade } \\
\text { e acessibilidade. }\end{array}$ \\
\hline 2002 & $\begin{array}{l}\text { Certificación } \\
\text { de Páginas } \\
\text { Web Sanitarias }\end{array}$ & Espanha & Espanhol & $\begin{array}{l}\text { Sites que } \\
\text { desejam obter } \\
\text { um selo de } \\
\text { qualidade }\end{array}$ & Certificação & $\begin{array}{l}\text { Audiência, usabilidade, } \\
\text { acessibilidade, } \\
\text { confidencialidade e privacidade, } \\
\text { transparência e honestidade, } \\
\text { credibilidade, política editorial, } \\
\text { meios de comunicação com os } \\
\text { usuários, autoria de conteúdo, } \\
\text { atualização, publicidade, } \\
\text { identidade e responsabilidade } \\
\text { do site, estrutura } \\
\text { organizacional, serviços e } \\
\text { profissionais. }\end{array}$ \\
\hline 2002 & $\begin{array}{l}\text { Guia de ética } \\
\text { para sites } \\
\text { de medicina } \\
\text { e saúde na } \\
\text { internet }\end{array}$ & Brasil & Português & $\begin{array}{l}\text { Provedores de } \\
\text { informação }\end{array}$ & $\begin{array}{l}\text { Código de } \\
\text { conduta }\end{array}$ & $\begin{array}{l}\text { Transparência, honestidade, } \\
\text { qualidade, consentimento livre } \\
\text { e esclarecido, privacidade, ética } \\
\text { médica, responsabilidade e } \\
\text { procedência. }\end{array}$ \\
\hline
\end{tabular}


Neste artigo, apresentamos um conjunto de critérios e indicadores úteis para a avaliação da qualidade da informação em sites de saúde. Nosso objetivo é que eles sirvam como um guia de orientação aos desenvolvedores de sites e provedores de informação. Nossa expectativa é que eles consigam, com este instrumento, construir ambientes virtuais de melhor qualidade e que despertem confiança no usuário.

Agrupamos os critérios, como indicadores de avaliação da qualidade da informação de saúde online, de acordo com as similaridades, complementaridades e áreas correspondentes. Assim criamos três dimensões de avaliação: conteúdo, técnica e design.

\section{Dimensão - Conteúdo}

Os critérios de abrangência, acurácia e inteligibilidade integram a nosso ver a dimensão que denominamos conteúdo. O critério de abrangência, contemplado pela maioria das iniciativas, pode ser medido pelo domínio que o site abarca sobre um tema ou problema de saúde. No caso de um site que contenha informações sobre uma doença transmissível, o ideal seria que ele apresentasse informações sobre o diagnóstico, a prevenção, as possibilidades de tratamento e não apenas sobre um destes aspectos. As informações devem ser detalhadas e advertir sobre os efeitos adversos do tratamento e dos medicamentos recomendados. Neste caso, recomendamos que sejam incluídos os seguintes indicadores:

- O site apresenta informações de diagnóstico

- O site apresenta informações de prevenção

- O site apresenta informações sobre possibilidades de tratamento

- O site apresenta informações sobre possíveis complicações do tratamento

- O site apresenta os benefícios e o desempenho dos tratamentos e/ou dos produtos

- O site apresenta contato de local para obter maiores informações sobre diagnóstico, tratamento e prevenção

Além da abrangência, a iniciativas de avaliação apresentadas anteriormente reiteram a importância da acurácia das informações publicadas. Ela é medida através do grau de concordância entre a informação oferecida online e a melhor evidência geralmente aceita pela prática médica ${ }^{11}$. Sugerimos, neste trabalho, que as afirmações disponibilizadas sobre os benefícios e/ou desempenho de tratamento, produto ou serviço de saúde respondam afirmativamente aos seguintes indicadores:

- O conteúdo está cientificamente fundamentado com base em estudos, pesquisas, protocolos, consensos ou prática clínica?

- O site apresenta as fontes que suportam as informações disponíveis?

- As fontes são de origem amplamente reconhecida e com boa reputação?

- O site possui um comitê científico?

A dimensão conteúdo inclui ainda o critério de intelegibilidade. Ele visa estabelecer o grau de compreensão do texto disponível. Ele avalia se o estilo de escrita está adequado ao público de destino. Na elaboração dos conteúdos, o gestor do site deve utilizar uma linguagem clara e acessível de forma que qualquer leitor possa compreender o que está sendo dito. Segundo recomendações de Eysenbach e colaboradores ${ }^{11}$, a informação dever ser segmentada conforme as características pessoais e as limitações do seu público. Apesar da sua importância, a intelegibilidade é o critério menos abordado pelas iniciativas apresentadas.

Para certificar se o conteúdo pode ser entendido pelo usuário para o qual a informação se destina, um site deverá procurar responder positivamente as seguintes questões: 
- A linguagem é adequada para o público de destino?

- As fontes do texto estão em tamanho adequado?

- É possível entender o propósito do site?

- O site não utiliza palavras técnicas ou jargão desconhecidos pelo público em geral?

- A página inicial do site tem poucas informações?

- Os textos são muito curtos e de rápida compreensão?

- O site apresenta mensagens claras de alertas ou de erros?

- As imagens na página ajudam a entender os textos?

\section{Dimensão - técnica}

A dimensão técnica contempla os critérios e indicadores que garantam credibilidade, segurança $e$ privacidade das informações acessíveis ao usuário. Inclui ainda aspectos éticos relativos à profissão do provedor de informação e perguntas relacionadas com o suporte técnico eficiente e adequado ao usuário. Além disso, deve ser verificado se o site possui o mínimo de interatividade com seus usuários.

Em relação à segurança e credibilidade, um site confiável deve apresentar nome e demais dados referentes do indivíduo ou instituição responsável por seu conteúdo. Além disso, deve constar a data de atualização e a política editorial que traduza as diretrizes que orientam a produção do conteúdo ${ }^{29}$. Segundo o Cremesp, um site deverá apresentar nome e logomarca, para o caso de pessoa jurídica, e currículo e credenciais, no caso de pessoa física. O endereço físico e o telefone do órgão devem estar em local visível em todas as páginas do site, localizado, preferencialmente, no rodapé. No caso de múltiplos endereços ou serviços, é recomendável a publicação de uma página relacionando os endereços de contato. Finalmente, o Conselho Regional de Medicina de São Paulo entende ser obrigatória a identificação dos profissionais que atuaram na construção daquelas informações ${ }^{30}$. O objetivo do site deve estar claro para quem o visita, seja de caráter informativo, educativo, comercial visando a venda de produtos ou a prestação de serviços de atenção médica personalizada, assessoria ou aconselhamento. Ele deve oferecer ferramentas que possibilitem ao usuário emitir opinião, apresentar suas dúvidas ou reclamações. Assim, o usuário poderá receber o retorno adequado a seus questionamentos, de forma ágil e apropriada ${ }^{30,31}$. Em suma, sugerimos que sejam relacionadas algumas questões técnicas para verificação da segurança e credibilidade de um site, tais como:

- Consta o autor da informação?

- As credenciais dos autores foram fornecidas?

- O site fornece os endereços de contato do autor?

- Consta o responsável do site?

- As credenciais dos responsáveis foram fornecidas?

- Consta a data de criação do site?

- Consta a data de atualização do site?

- Consta a data de criação do conteúdo?

- Consta a data de atualização em todas as páginas/conteúdos do site?

- O site conta com revisão editorial?

- Consta no site a política editorial?

- O propósito do site é apresentado?

- O objetivo do site está claro?

- O site menciona o público para o qual as informações se destinam?

- O site foi certificado por alguma instituição credenciadora? 
O aspecto comercial também nos parece fundamental. Diversos sites de saúde estão a serviço exclusivamente dos patrocinadores, geralmente empresas de produtos e equipamentos médicos, além da indústria farmacêutica. Estas empresas muitas vezes, interferem no conteúdo e na linha editorial dos sites, pois estão interessadas em comercializar seus produtos ${ }^{30}$. Por esta razão, a nosso ver, deverá ser indicado explicitamente se a publicidade é uma das fontes de renda do site. Além disso, os responsáveis deverão fornecer uma breve descrição da política de divulgação adotada. Os anúncios e outros materiais promocionais deverão ser apresentados de tal maneira e em tal contexto, que permita que o usuário os identifique ${ }^{32}$. Sendo assim, entendemos que devam ser incluídos, nesta dimensão, os seguintes indicadores:

- O apoio e parceria financeira é mencionado

- Os anúncios publicitários são claramente identificados

- Há distinção entre anúncios e o conteúdo

- O site possui política de publicidade

A segurança, a credibilidade e a preocupação com o lado comercial se juntam ao aspecto ético do gestor e provedor de informação. No Brasil, o Cremesp determina que o profissional responsável pelos sites responderá pela infração ética junto ao Conselho de Medicina "se a ação, omissão, conduta inadequada, imperícia, negligência ou imprudência, via internet, produzirem dano à vida ou agravo à saúde do indivíduo" . Em relação à ética sugerimos que seja verificado se:

- Os profissionais de saúde estão cumprindo os preceitos éticos e legislativos da profissão?

- Os aconselhamentos em saúde são prestados por profissionais qualificados?

- O site notifica a necessidade de consulta médica?

- Os parceiros estão de acordo com os princípios éticos adotados pelo site?

A dimensão técnica não poderia deixar de abordar o problema da privacidade do cidadão. Diversos sites solicitam informações de seus usuários ao acessar seu conteúdo. Quando for este o caso, devem ser honrados os requisitos mínimos de privacidade sobre a informação pessoal obtida, de acordo com as legislações vigentes de cada país, conforme indica o AMA Guidelines da American Medical Association. O site deve solicitar a permissão do usuário antes de coletar qualquer informação pessoal. Ele deve informar, com clareza, os motivos da coleta, assim como, a finalidade de seu armazenamento e os riscos potenciais de sua utilização. Para tanto, o site deve pedir o consentimento ao usuário antes do procedimento ser realizado. Por esta razão, os termos de uso e as políticas de segurança e privacidade devem estar escritos de forma clara, objetiva e ter fácil visualização. No caso de transações comerciais, medidas de segurança devem ser seguidas para evitar que terceiros tenham acesso a dados pessoais para outra finalidade que não seja a proposta pelo site ${ }^{31}$. Resumindo, a nosso ver, os indicadores que merecem ser verificados em relação à privacidade, com respostas afirmativas às seguintes questões, são:

- O site solicita autorização do usuário no momento da coleta de seus dados pessoais?

- O site informa a finalidade da coleta desses dados?

- O sigilo de dados pessoais dos usuários está assegurado através de uma política de segurança e privacidade?

- O site informa o propósito da coleta das informações pessoais?

- O site possui sistema de proteção e segurança como o de criptografia de dados?

- O usuário tem a possibilidade de apagar seus dados registrados no site? 
Credibilidade e privacidade integram esta segunda dimensão assim como a interatividade. De acordo com a iniciativa Criteria for Assessing the Quality of Health Information on the Internet da Agency for Health Care Policy and Research (AHRQ), dos Estados Unidos, interatividade é o "mecanismo de retorno da informação, fórum de discussão"33. Segundo o Net Scoring, ela pode ser proporcionada por intermédio dos mecanismos de contato, fóruns, chats e avisos aos usuários.

O desempenho de qualquer sistema interativo melhora quando os procedimentos necessários ao cumprimento da tarefa são compatíveis com as características psicológicas, culturais e técnicas do usuário, e os procedimentos e as tarefas são organizados de acordo com as suas expectativas e costumes ${ }^{34}$. Os usuários que buscam informações sobre saúde desejam compartilhar suas aflições e experiências. Eles têm curiosidades e interesses singulares. Muitas vezes procuram auxílio e orientação dos provedores do site ou de participantes das redes sociais associadas a ele. Este público reúne indivíduos acometidos por diversas doenças, em especial as crônicas, raras ou com estigma. Eles buscam ansiosamente respostas para seus questionamentos sobre um estado de saúde ou diagnóstico. A interatividade, e o devido suporte ao usuário, devem ser incluídos, a nosso ver, como indicadores de qualidade da dimensão técnica. Algumas questões nos parecem pertinentes neste sentido como:

1. O site possui ferramentas interativas (fórum, blog, chat, redes sociais, comentários)?

2. Existem regras estabelecidas para a utilização dessas ferramentas?

3. Estas ferramentas possuem uma pessoa responsável (moderador)?

4. O site apresenta as credenciais/qualificações do moderador?

5. O moderador está apto a responder dúvidas e questionamentos sobre o assunto abordado?

6. O site fornece contatos do usuário com o webmaster?

7. O site oferece FAQ (Perguntas mais frequentes) para os usuários?

8. O site oferece tutoriais de ajuda para o usuário?

9. O site possui fale conosco?

10. As questões dos usuários são respondidas em tempo ágil?

11. O site avalia a satisfação do usuário quanto ao seu atendimento ou conteúdo?

\section{Dimensão - Design}

A maioria das iniciativas de avaliação, mencionadas anteriormente, concentram sua atenção no conteúdo da informação. Verificar se as informações disponíveis em um site estão em conformidade com a dimensão de conteúdo não nos parece suficiente. Segundo Castiel e Vasconcelos-Silva ${ }^{7}$, é necessário compreender o ambiente dinâmico da web e seu público com suas características sociais, cognitivas e culturais.

A dimensão design confere critérios que indicam a facilidade de uso, de navegação e acessibilidade de acordo com as necessidades e expectativas dos usuários, reunindo critérios de usabilidade e acessibilidade. A usabilidade refere-se aos aspectos de interface, como a navegação, velocidade, compatibilidade com navegadores e disponibilidade de recursos para pessoas com poucos recursos tecnológicos. Um site de qualidade será aquele que poderá ser acessado independentemente de equipamentos, programas e formatos específicos $^{35}$. A acessibilidade refere-se aos critérios para garantir que pessoas com deficiências visuais, auditivas e motoras possam acessar a informação sem dificuldade.

Cabe destacar que um site com informações confiáveis e seguras, corre o risco de ter baixos índices de usabilidade ou ser inacessível para pessoas com deficiências. Recomendamos, neste artigo, que os desenvolvedores de sites consultem a "Cartilha de Usabilidade" 36 e o "e-MAG Modelo de Acessibilidade em Governo Eletrônico" ${ }^{37}$. Estes são dois documentos de referência publicados pelo Ministério de Planejamento 
em 2010 e 2011. Os indicadores de usabilidade e acessibilidade que se seguem foram retirados destes documentos, a saber:

1. Constam logotipos, atalhos e caixas de busca visíveis em todas as páginas e sempre no mesmo lugar?

2. É fácil de navegar no site?

3. O design do site é agradável e limpo?

4. O site possui links de retornos em todas as páginas?

5. Existe ferramenta de busca no site?

6. A ferramenta de busca está presente em todas as páginas?

7. O site apresenta a opção de busca avançada depois da busca simples?

8. Os resultados da busca são pertinentes?

9. Consta o mapa do site?

10. No caso de baixa de arquivos, os links estão acompanhados de descrições claras e precisas sobre o seu conteúdo, tamanho e formato?

11. O site possui uma seção de destaque para informações mais recentes?

12. Os links do site funcionam?

13. As categorias de links são claras e bem definidas?

14. O site disponibiliza atalhos de teclado?

15. O site possui conteúdo alternativo para imagens?

16. São fornecidas descrições para as imagens da página, utilizando-se o atributo alt?

17. As páginas são leves e carregam rapidamente? O site pode ser acessado em mais de um Browser (navegador)?

18. O site pode ser acessado em mais de um dispositivo (celular, tablet)?

19. O site possui mecanismos para aumentar a fonte?

20. O site disponibiliza o recurso de barra de acessibilidade?

21. O site disponibiliza o mesmo conteúdo em vários formatos (texto, áudio e vídeo)?

22. Os documentos em pdf também são disponibilizados em HTML?

\section{Conclusão}

As informações obtidas na internet, muitas vezes, pela sua proporção e heterogeneidade, podem ser de baixa qualidade, ter origem duvidosa, ser insuficiente, estar desatualizada e ter pobre embasamento científico $^{14,18-19-20}$.

As iniciativas para avaliação da qualidade das informações sobre saúde em sites brasileiros são mínimas diante deste oceano informacional. No Brasil, não há notícia de órgão público ou privado que certifique sites de saúde. Iniciativa desta natureza implicaria o envolvimento de profissionais de saúde, de informação e usuários. Cada um destes segmentos é dotado de uma perspectiva própria de avaliação. Neste sentido, este artigo reuniu três dimensões para avaliação da qualidade da informação em sites de saúde. Com isso, pretende-se, de certa forma, incentivar a atuação, por parte de órgão competente do governo brasileiro, de modo a criar um selo de qualidade para os sites governamentais de saúde no Brasil. Visa-se, ainda, orientar desenvolvedores de ambientes virtuais para produzirem sites com critérios mínimos de qualidade. Almejase, finalmente, orientar usuários da internet a conferirem a qualidade de conteúdos recuperados na web.

A nosso ver, é urgente a criação de um selo que seja facilmente identificado pelos usuários. Um selo que torne público critérios e indicadores utilizados. Os sites certificados constituiriam um catálogo nacional de referência para os usuários, disponível para o acesso público através de um portal temático dedicado exclusivamente às questões de saúde, com linguagem adequada para o público leigo. 


\section{Referências}

1. Recuero, R. Redes sociais na internet. Porto Alegre: Sulina, 2009. (Coleção Cibercultura). 191 p.

2. Cubas, MR; Felchner, PCZ. Análise das fontes de informação sobre os autoexames da mama disponíveis na Internet. Ciênc. Saúde Coletiva, Abr 2012, 17(4): 965-970.

3. Silva EV; Castro, LLC; Cymrot, R. Tratamento farmacológico da obesidade em páginas da Internet brasileira: análise dos critérios técnicos de qualidade. Rev Ciên Farm Básica Apl. 2008, 29(2): 161-7.

4. Silva, L; Mello JR, JF; Mion, O. Avaliação das informações sobre rinite alérgica em sites brasileiros na rede mundial de computadores (Internet). Rev Bras Otorrino., 2005, 71(5): 590-597.

5. Garbin, HBR; Pereira Neto, AF; Guilam, MCR. A internet, o paciente expert e a prática médica: uma análise bibliográfica, Brasil. Interface. 2008;12(26): 579-588.

6. Moraes, IHS. Política, tecnologia e informação em saúde: a utopia da emancipação. Salvador: Casa da Qualidade; 2002.

7. Castiel, LD; Vasconcellos-Silva, PR. Internet e o auto-cuidado em saúde: como juntar os trapinhos? História, Ciências, Saúde - Manguinhos. 2002;9 (2): 291-314.

8. Lopes, IL. Novos paradigmas para avaliação da qualidade da informação em saúde recuperada na Web. Ciência da Informação. 2004;33(1): 81-90, 2004.

9. Fogg, BJ et al. Stanford-Makovsky web credibility study 2002: investigating what makes web sites credible today - a research report by the Stanford Persuasive Technology Lab \& Makovsky \& Company. [S.I.]: Stanford University [Internet]. 2002. [cited 2012 Mar 15] Available from: www.webcredibility.org

10. Mostafa, S. P.; Terra, M. As fontes eletrônicas de informação: novas formas de comunicação e de produção do conhecimento. SP em Persp. 1998 out./dez., 12(4): 1-12.

11. Eysenbach, G. et al. Empirical studies assessing the quality of health information for consumers on the World Wide Web: a systematic review. Journal Am Med Assoc. 2002, 287(20): 2691-2700.

12. Risk, A; Dzenowagis, J. Review of internet health information quality initiatives. Journal Med Internet Res. 2001, 3(4): 28.

13. Souza, CLN; Luz, ZP; Rabello, A. Análise da informação sobre a leishmaniose visceral disponível em portais brasileiros da rede mundial de computadores: internet. Rev Soc Bras Med Trop. 2008 41(4): 352357.

14. Lopes, IL. Proposta de critérios de qualidade para avaliação da informação em saúde recuperada nos sites brasileiros da World Wide Web [teses]. Brasília: Universidade de Brasília; 2006.

15. Lopes, IL. Estudos sobre qualidade da informação em saúde na web e a visão de entidades de classe brasileiras. Tempus: Actas de Saúde Coletiva. 2008, 2: 1-23.

16. Leite F; Correia, A. Quality evaluation of websites with information on childhood dental caries in portuguese language. Rev Odonto Ciên. 2011, 26(2): 116-120.

17. Silva, RQ; Gubert, MB. Qualidade das informações sobre aleitamento materno e alimentação complementar em sites brasileiros de profissionais de saúde disponíveis na internet. Rev Bras Saúde Mat Inf. 2010, 10(3): 331-340.

18. Malafaia, G; Castro, ALS; Rodrigues, ASL. A qualidade das informações sobre doenças disponíveis em websites brasileiros: uma revisão. Arq Bras Ciên Saúde. 2011, 36(2): 72-8.

19. Silveira, PCM; Costa, AES; Lima, CC. Gagueira na web: qualidade da informação. CEFAC. 2012, 14(3):430-437.

20. Silberg WM, Lundberg GD, Musacchio RA. Assessing, controlling and assuring the quality of medical information on the Internet. J Am Med Assoc. 1997; 277(15):1244-5.

21. Jadad, AR; Gagliardi, A. Rating health information on the internet: navigating to knowledge or to Babel? Journal of American Medical Association. 1998. 279(8): 611-614.

22. Gagliardi, A; Jadad, A. Examination of instruments used to rate quality of health information on the internet: chronicle of a voyage with an unclear destination. British Medical Journal. 2002, 321: 569-573. 
23. Centro Latino-Americano e do Caribe de Informação em Ciências da Saúde, et al. Metodologia LIS: critérios para seleção de fontes de informação em saúde disponíveis na Internet. 2002. 2. rev. São Paulo: Bireme; OPS; OMS.

24. Moretti, FA; Oliveira, VE; Silva, EMK. Acesso a informações de saúde na internet: uma questão de saúde pública? Rev Assoc Méd Bras. 2012, 58(6): 650-658.

25. Winker, MA et al. Guidelines for medical and health information sites on the internet: principles governing AMA web sites. Journ Am Med Assoc. 2000, 283(12): 1600-6.

26. Agency for Healthcare Research and Quality. Assessing the Quality of Internet Health Information. Agency for Healthcare Research and Quality [Internet].1999 jun. [cited 2015 Jan 21]. Disponível em: http://archive.ahrq.gov/research/data/infoqual.html

27. winker, ma. et al. Guidelines for ama web sites. American Medical Association [Internet]. 2000 mar [cited 2015 Jan 19] 12:22-29. Available from: http://www.ebm.lib.ulg.ac.be/prostate/pdf/siteweb.pdf

28. Internet Healthcare Coalition. E-health Code of Ethics[Internet]. 2000 [cited 2015 Jan 19]. Available from: http://www.ihealthcoalition.org/ehealth-code-of-ethics.

29. Utilization Review Accreditation Commission. Accreditation Programs. Utilization Review Accreditation Commission [Internet]. [date unknown]. [cited 2015 Jan 19] Available from: https://www.urac.org/ accreditation-and-measurement/accreditation-programs/

30. Euro Health net. Health Literacy and Internet: recommendations to promote health literacy by the means of the internet. Euro Health Net [Internet]. 2005 Apr [cited 2015 Jan 19]. Available from: http:// eurohealthnet.eu/sites/eurohealthnet.eu/files/publications/pu 8.pdf

31. Discern Project. Welcome to discern [Internet]. 1997. [cited 2015 Jan 19] Available from: http://www. discern.org.uk/ Acesso em 19 jan. 2015.

32. France Centrale Santé. Net Scoring $\mathbb{R}$ : critères de qualité de l'information de santé sur l'Internet. [cité 19 jan 2015] Disponible dans : http://www.chu-rouen.fr/netscoring/

33. Hon Foundation. Código de conduta para sites web em Medicina e Saúde (HONcode). 1999. [citado 19 jan 2015]. Disponível em: http://www.hon.ch/HONcode/Portuguese

34. Web Mèdica Acreditada. Programa de Certificación y Calidad de páginas Web de Salud. [data desconhecida]. [citado 19 jan 2015] Disponível em: http://wma.comb.es/es/home.php

35. São Paulo (Estado). Conselho Regional de Medicina do Estado de São Paulo. Guia de ética para sites de medicina e saúde na internet [ Internet]. [citado 19 jan 2015] Disponível em: http://www.cremesp.org. $\underline{\mathrm{br} / \text { ? siteAcao }=\text { Publicacoes\&acao }=\text { detalhes capitulos\&cod capitulo }=26}$

36. Brasil. Ministério do Planejamento, Orçamento e Gestão. Secretaria de Logística e Tecnologia da Informação. Padrões Web em Governo Eletrônico: Cartilha de Usabilidade. Ministério do Planejamento, Orçamento e Gestão, Secretaria de Logística e Tecnologia da Informação [Internet].2010. [citado 19 mar 2015]. Disponível em: <http://www.governoeletronico.gov.br/acoes-e-projetos/padroes-brasil-e-gov>

37. Brasil. Ministério do Planejamento, Orçamento e Gestão. Secretaria de Logística e Tecnologia da Informação. e-MAG Modelo de Acessibilidade em Governo Eletrônico [Internet]. 2011. [citado 19 mar 2015]. Disponível em: http://www.governoeletronico.gov.br/acoes-e-projetos/e-MAG> 